\title{
SURFACE CHEMICAL STATES OF HETEROEPITAXIAL NITRIDE FILMS ON SAPPHIRE BY METALORGANIC CHEMICAL VAPOR DEPOSITION
}

\author{
K. LI \\ Chartered Semiconductor Manufacturing Ltd. \\ 60 Woodlands, Industrial Park D, Street 2, Singapore 738406 \\ Z. C. FENG* and C.-C. YANG \\ Graduate Institute of Electro-Optical Engineering and \\ Department of Electrical Engineering, National Taiwan University \\ Taipei 106-17, Taiwan, ROC \\ *zcfeng@cc.ee.ntu.edu.tw \\ J. LIN \\ Department of Physics, National University of Singapore, Singapore 119260
}

\begin{abstract}
Surface chemical states of GaN, AlGaN and $\mathrm{InGaN}$ by metalorganic chemical vapor deposition, and the influence of different dopants are studied with X-ray Photoelectron Spectroscopy (XPS). The results show that for most of the samples the $\mathrm{N}$ 1s peak can be fitted with a dominant $\mathrm{GaN}$ peak and a small $\mathrm{N}-\mathrm{H}$ peak, while $\mathrm{Ga} 3 \mathrm{~d}$ can be deconvoluted into three peaks from elemental $\mathrm{Ga}$, $\mathrm{GaN}$ and $\mathrm{Ga}_{2} \mathrm{O}_{3}$. Si-doping appears to have small influence on the surface chemical states of GaN while the influence of Mg-doping appears larger. In addition to a change in the component intensities, Mgdoping also causes the $\mathrm{N}$ 1s and Ga $3 \mathrm{~d}$ peaks to broaden. The ternary AlGaN sample shows aluminum surface segregation, while the undoped InGaN shows indium surface deficiency.
\end{abstract}

Keywords: Metalorganic chemical vapor deposition; X-ray photoelectron spectroscopy; GaN.

\section{Introduction}

GaN and its ternary alloys InGaN and AlGaN are the main materials for the bluegreen light-emitting optoelectronic and other electronic devices. GaN-based Light Emitting Diodes (LEDs) are now commercially available and marvelous progress has been made in recent years. ${ }^{1-3}$ Metalorganic Chemical Vapor Deposition (MOCVD) technology has been widely employed in research and industry production for these GaN-based optoelectronic and electronic materials and devices currently. ${ }^{1,3}$ However, many technological and scientific barriers still exist in this materials system. The most dominant ones are stoichiometric nitrogen incorporation, $p$-type 
doping, and the availability of lattice- and thermally-matched substrates. Due to the use of large amounts of various organic and inorganic gases in the chemical reaction chamber, the surface chemical states of grown materials are affected by many factors. It has been found that the composition and structure of III-V nitride films strongly influence their optoelectronic properties. Doping can also change the strength of light emitted. The fabricated semiconductor devices are sensitive to surface properties.

X-ray Photoelectron Spectroscopy (XPS) is a sensitive surface science analytical technology and has been used in recent years for GaN and related materials; to fingerprint the surface composition and morphology, ${ }^{4,5}$ to study on the Mg-doped $p$-GaN,${ }^{6,7}$ surface oxides, ${ }^{8}$ surface passivation, ${ }^{9}$ selective etching, ${ }^{10}$ high temperature annealing, ${ }^{11}$ and ohmic contacts to $n$-GaN ${ }^{12,13}$ and $p$-GaN. ${ }^{14}$

In this report, the surface chemical states of MOCVD grown GaN, AlGaN and InGaN on sapphire substrate, and the influence of different dopants have been studied systematically by X-ray Photoelectron Spectroscopy (XPS).

\section{Experimental Details}

The samples were grown using an EMCORE MOCVD system with the vertical growth configuration and high speed rotating disk. The chemical precursors used for gallium, indium, aluminum and nitrogen are trimethylgallium ( $\left.\mathrm{TMG}, \mathrm{Ga}\left(\mathrm{CH}_{3}\right)_{3}\right)$, trimethylindium (TMIn, $\mathrm{In}\left(\mathrm{CH}_{3}\right)_{3}$ ), trimethylaluminum ( $\left.\mathrm{TMAl}, \mathrm{Al}\left(\mathrm{CH}_{3}\right)_{3}\right)$, and ammonia $\left(\mathrm{NH}_{3}, 100 \%\right)$, respectively. The dopant precursors for magnesium and silicon are biscyclopentadienyl magnesium $\left(\mathrm{Cp}_{2} \mathrm{Mg}\right)$ and silane $\left(\mathrm{SiH}_{4}\right)$, respectively. To overcome the negative effect of significant lattice mismatch between the GaN overlayer and the sapphire substrate, a $200 \AA \sim 300 \AA$ thick GaN buffer layer, amorphous or polycrystalline, was first grown on the sapphire substrate at $530^{\circ} \mathrm{C}$. The temperature was subsequently raised to $1040^{\circ} \mathrm{C}$ and the GaN epilayer was grown to a thickness of about $2 \mu \mathrm{m}$. The ternary films InGaN and AlGaN were further deposited on the $2 \mu \mathrm{m}$ thick GaN layers to a thickness of about $1000 \AA$. The growth temperatures used for InGaN and AlGaN were $800^{\circ} \mathrm{C}$ and $1050^{\circ} \mathrm{C}$, respectively. These epitaxial layers were measured by X-ray diffraction/TEM, with single crystalline structure (not shown here).

XPS studies were carried out in a VG ESCALAB Mk II system using a $120 \mathrm{~W}$ $\mathrm{Mg} \mathrm{K} \alpha$ X-ray source. The XPS spectra were collected at a photoelectron take-off angle of $75^{\circ}$ with respect to the sample surface. The Constant Analyzer Energy (CAE) mode was used with an analyzer pass energy of $50 \mathrm{eV}$ for wide scans and $20 \mathrm{eV}$ for narrow scans. All binding energies were referenced to the $\mathrm{C} 1 \mathrm{~s}$ peak of adventitious carbon at $284.8 \mathrm{eV}$. Quantification was done using tabulated semiempirical Atomic Sensitivity Factors (ASFs), ${ }^{15}$ and checked with standards.

Three pieces of GaN/sapphire samples in this paper were also studied by Hall measurements. The carrier concentrations were determined to be less than $5 \times 10^{16} \mathrm{~cm}^{-3}$ (n-type) for the undoped GaN, $\sim 2 \times 10^{18} \mathrm{~cm}^{-3}$ for the $n$-type 
Si-doped one, and $\sim 8 \times 10^{16} \mathrm{~cm}^{-3}$ for the $p$-type Mg-doped GaN (after annealing), respectively.

\section{Results and Discussion}

\subsection{GaN: Undoped and Si-/Mg-doped}

Figure 1 compares the N 1s and Ga 3d peaks of the undoped GaN, $p$-type Mg-doped GaN, and $n$-type Si-doped GaN. All the N 1s peaks can be fitted with two components corresponding to $\mathrm{GaN}^{16}$ and $\mathrm{N}-\mathrm{H}_{x}(x<3)^{17}$ bonding, respectively. The Ga $3 \mathrm{~d}$ peaks can be fitted with three components resulting from elemental Ga, GaN and $\mathrm{Ga}_{2} \mathrm{O}_{3}$, respectively. ${ }^{16}$ The existence of carbon and oxygen on the surface is due to the ambient exposure.

As shown in Fig. 1(b) for the Si-doped $n$-GaN, the influence of Si-doping on the surface chemical states appears to be small because there is neither peak broadening nor peak position shift. However, the Si-doped sample has a lower oxide component

$\mathrm{N} 1 \mathrm{~s}$

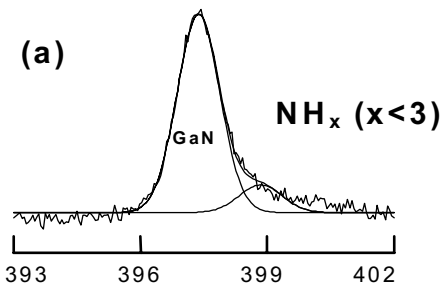

(b)
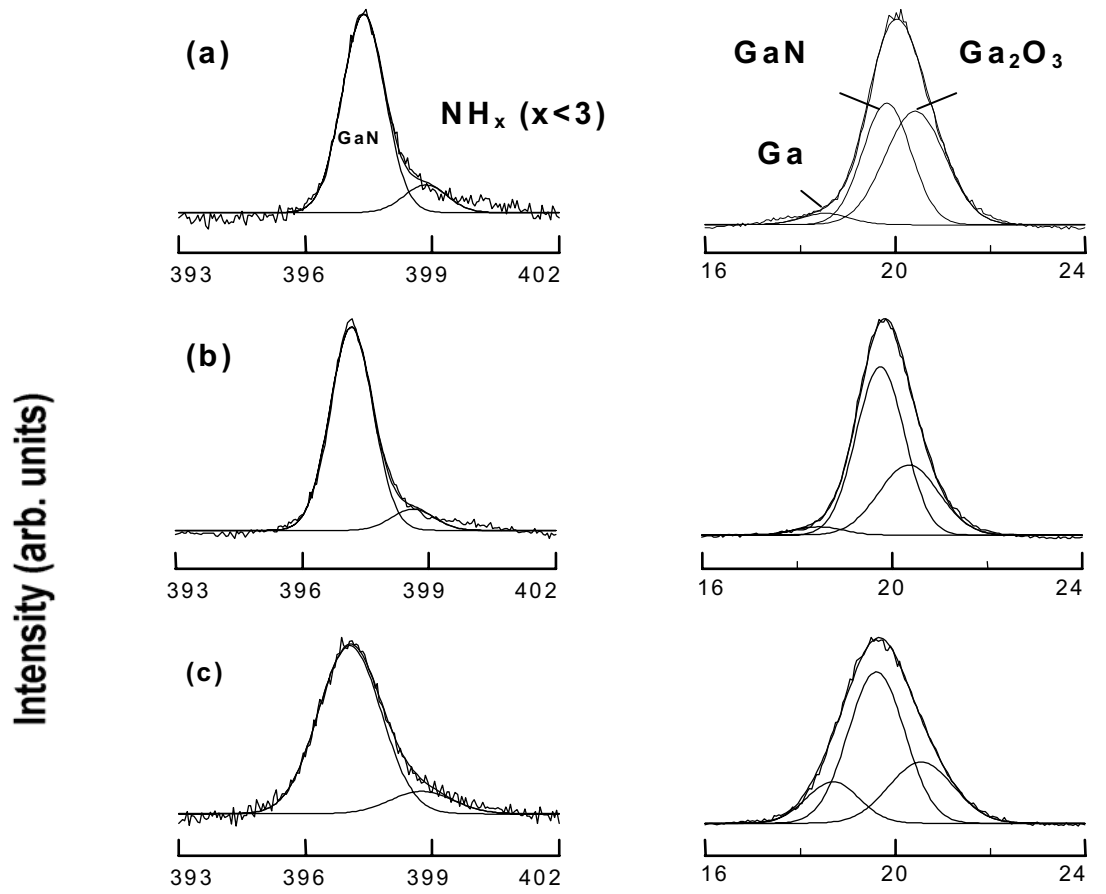

\section{Binding Energy (eV)}

Fig. 1. XPS narrow scans of N 1s, Ga 3d peaks from the GaN epilayers of (a) undoped GaN; (b) $n$-type Si-doped GaN; and and (c) p-type Mg-doped GaN. 
for the Ga 3d peak as compared with that of undoped GaN, and a lower elemental Ga percentage $(2.1 \%)$. In addition, the Si-doped sample is more gallium deficient at the surface $(\mathrm{Ga} / \mathrm{N} \sim 0.6)$.

Compared with Si-doping, $\mathrm{Mg}$ p-type doping appears to have a significant influence on the surface chemical states (Fig. 1(c)). Both the N 1s and Ga 3d peaks are broader than the corresponding peaks in the undoped GaN. For example, the FWHMs of the GaN and $\mathrm{N}-\mathrm{H}_{x}$ components of the $\mathrm{N}$ 1s peak in the Mg-doped GaN are both $1.75 \mathrm{eV}$, larger than the value of $1.2 \mathrm{eV}$ for the undoped GaN. Upon $p$-type $\mathrm{Mg}$-doping, the percentage of elemental gallium at the surface increases by about $12 \%$, from $3.2 \%$ for the undoped GaN to $15.5 \%$ of the total surface Ga content, while the fraction of $\mathrm{Ga}_{2} \mathrm{O}_{3}$ decreases from $52.2 \%$ to $24.2 \%$.

The observation that the $\mathrm{Ga}_{2} \mathrm{O}_{3}$ component in the $\mathrm{Ga} 3 \mathrm{~d}$ peak from undoped $\mathrm{GaN}$ is larger than those from $\mathrm{Si}-/ \mathrm{Mg}$-doped samples, can be understood qualitatively. The surface from $\mathrm{Si}$-/Mg-doped samples should have less free-hanging unbonded states, due to doping, in comparison with undoped GaN. This can lead to the larger degree of surface oxidation from undoped GaN than that from Si-/Mg-doped GaN. The filling or partial filling of the surface free-hanging unbonded states due to doping may also modify the surface energy. In our TEM and AFM study of differently doped GaN (not shown here), we find that the dopants ( $\mathrm{Mg}$ and $\mathrm{Si}$ ) can cause significant change in dislocation core structures and surface morphology due to the change of surface energy caused by the dopants. Therefore, it is suggested that the surface energy change caused by Mg-doping is responsible for the change in the surface chemical states. The presence of $\mathrm{Mg}$ at the surface is also detected with XPS, and the atomic concentration of $\mathrm{Mg}$ is found to be about $0.8 \%$ of the total surface atomic composition. The $\mathrm{Ga} / \mathrm{N}$ ratio drops from 1.2 without doping to 0.8 after doping.

\subsection{AlGaN: Updoped}

The $\mathrm{N}$ 1s, Ga 3d and $\mathrm{Al} 2 \mathrm{p}$ narrow scans of $\mathrm{Al}_{x} \mathrm{Ga}_{1-x} \mathrm{~N}(x \sim 0.025)$ and their respective peak fittings are shown in Fig. 2. Comparing the peak fittings of $\mathrm{N} 1 \mathrm{~s}$ and $\mathrm{Ga} 3 \mathrm{~d}$ for the AlGaN sample in this figure with those for the undoped GaN sample (Fig. 1(a)), it can be seen that there are no discernible changes in either peak position (binding energy) or peak width (FWHM) for the corresponding peaks. However, some variations in the component intensities are observed, especially for the Ga 3d peak. There is a small amount of elemental Ga present at the surface $(\sim 3 \%)$. The percentage of the oxide component is about $43 \%$, which is less than that of the GaN sample $(\sim 52 \%)$. The results suggest that the presence of $\mathrm{Al}$ can also influence the surface oxidation of GaN. This is similar to the case of AlGaAs in which the oxidation due to $\mathrm{Al}$ is much faster than that in GaAs.

The $\mathrm{Al} 2 \mathrm{p}$ peak can be fitted with two components. The component at the binding energy of $73.8 \mathrm{eV}$ is assigned to AlGaN. Although the binding energy of $\mathrm{Al} 2 \mathrm{p}$ from $\mathrm{AlGaN}$ has not previously been reported, the assignment appears 


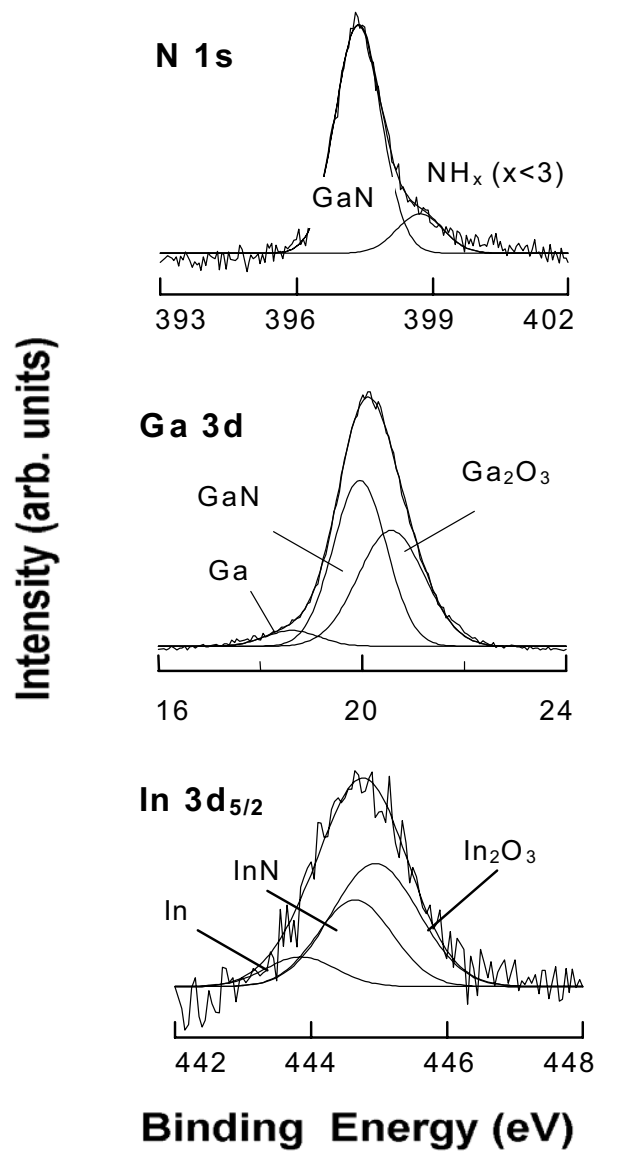

Fig. 2. XPS narrow scans of $\mathrm{N} \mathrm{1s,} \mathrm{Ga} 3 \mathrm{~d}$ and $\mathrm{Al} 2 \mathrm{p}$ peaks from the undoped AlGaN.

reasonable since AlGaAs has an $\mathrm{Al} 2 \mathrm{p}$ binding energy of about $73.6 \mathrm{eV}{ }^{18}$ The other component located at $74.7 \mathrm{eV}$ is assigned to either or both of $\mathrm{AlN}$ and $\mathrm{Al}_{2} \mathrm{O}_{3} .{ }^{16}$ The $\mathrm{Al}$ atomic composition at the surface is found to be about $2.6 \%$ of the total $\mathrm{Al}, \mathrm{Ga}$ and $\mathrm{N}$ surface content, implying that the $x$ value at surface is $\sim 0.052$ (note that the atomic composition should be doubled when converted into $x$ value for $\mathrm{Al}_{x} \mathrm{Ga}_{1-x} \mathrm{~N}$ under stoichiometric conditions, i.e. $\left.[\mathrm{Al}+\mathrm{Ga}]:[\mathrm{N}]=1: 1\right)$. This is appreciably larger than the bulk value of 0.025 , indicating the segregation of $\mathrm{Al}$ atoms in the near surface region.

\subsection{InGaN: Undoped}

The $\mathrm{N} 1 \mathrm{~s}$, Ga $3 \mathrm{~d}$ and $\mathrm{In} 3 \mathrm{~d}_{5 / 2}$ peaks of the undoped $\mathrm{In}_{x} \mathrm{Ga}_{1-x} \mathrm{~N}(x \sim 0.12)$ are shown in Fig. 3. The fitting of $\mathrm{N} 1 \mathrm{~s}$ and Ga $3 \mathrm{~d}$ peaks are almost the same as in Fig. 1(a) for the undoped GaN in terms of peak position and FWHM. However, there are some variations in the relative component intensities, especially for the Ga 


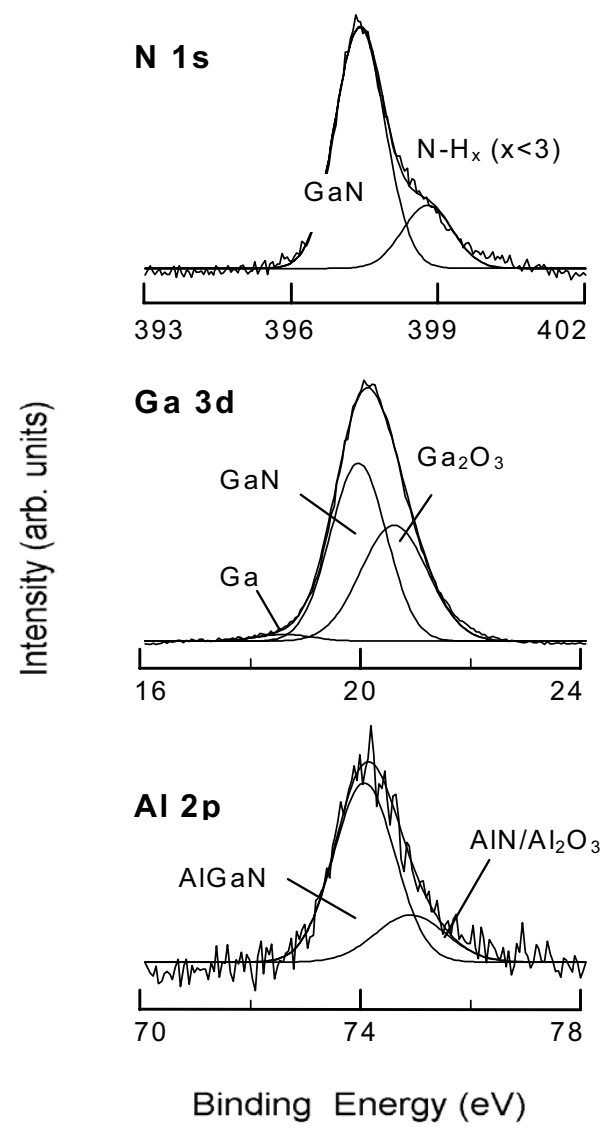

Fig. 3. XPS narrow scans of $\mathrm{N} 1 \mathrm{~s}, \mathrm{Ga} 3 \mathrm{~d}$ and $\mathrm{In} 3 \mathrm{~d}_{5 / 2}$ peaks from the undoped InGaN.

3d peak. The relative component intensities of Ga 3d for the InGaN sample, which are $4 \%$ elemental $\mathrm{Ga}, 45 \% \mathrm{Ga}_{2} \mathrm{O}_{3}$ and $51 \% \mathrm{GaN}$, respectively. The In $3 \mathrm{~d}_{5 / 2}$ peak can be deconvoluted into three components at $443.8 \pm 0.1 \mathrm{eV}$ for metallic indium, $444.4 \pm 0.1 \mathrm{eV}$ for InN, and $445.0 \pm 0.1 \mathrm{eV}$ for $\mathrm{In}_{2} \mathrm{O}_{3}$ with the FWHMs of $1.2 \mathrm{eV}$, $1.3 \mathrm{eV}$ and $1.5 \mathrm{eV}$, respectively. ${ }^{16}$ From the integrated peak area weighted by the XPS sensitivity factor, the surface atomic content of indium is found to be only about 0.022 , implying a surface $x$ value of about 0.04 . This is much lower than the bulk $x$ value of 0.12 , demonstrating the surface deficiency of indium.

\section{Conclusion}

For most of the samples, the $\mathrm{N}$ 1s peaks can be fitted with a dominant GaN peak at the binding energy of $397.2 \pm 0.2 \mathrm{eV}$ and a small $\mathrm{N}-\mathrm{H}_{x}$ peak at $398.5 \pm 0.2 \mathrm{eV}$, while $\mathrm{Ga} 3 \mathrm{~d}_{5 / 2}$ peak can be deconvoluted into three peaks, i.e. elemental $\mathrm{Ga}$ at $18.5 \pm 0.1 \mathrm{eV}, \mathrm{GaN}$ at $19.7 \pm 0.1 \mathrm{eV}$ and $\mathrm{Ga}_{2} \mathrm{O}_{3}$ at $20.4 \pm 0.1 \mathrm{eV}$. The influence 
of Si-doping on surface chemical states appears to be small, and therefore, it does not cause significant peak broadening. In contrast, Mg-doping appears to have a more significant influence on the surface chemical states of GaN. In addition to the variation in the component intensities for the Ga $3 \mathrm{~d}$ peak, significant peak broadening is observed. Moreover, aluminum surface segregation is observed in the undoped AlGaN sample, and the undoped InGaN sample is In-deficient. Further studies on the surface chemical states from GaN with different doping levels, ternary InGaN and AlGaN with large range of compositions and dopings, the dependence on the growth conditions such as growth temperature and growth rate, and penetrating mechanism investigation will be carried out in the near future; to get the whole spectrum of the knowledge on the chemical states of these important III-Nitrides and to enhance their applications.

\section{References}

1. S. Nakamura, S. Pearton and G. Fasol, The Blue Laser Diodes - The Complete Story (Springer, Berlin, 2000).

2. M. O. Mansreh and H. X. Jiang (eds.), III-Nitride Semiconductors: Optical Properties II, Vol. 14, Series of Optoelectronic Properties of Semiconductors and Superlattices, ed. M. O. Manasreh (Taylor \& Francis Books, New York, 2002).

3. M. O. Mansreh and I. Ferguson (eds.), III-Nitride Semiconductors: Growth, Vol. 19 ibid (2003).

4. M. Losurdo, P. Capezzuto, G. Bruno, G. Leo and E. A. Irene, J. Vac. Sci. Technol. A 17, 2194 (1999).

5. K. M. Tracy, W. J. Mecouch, R. F. Davis and R. J. Nemanich, J. Appl. Phys. 94, 3163 (2003).

6. Y.-J. Lin, C.-D. Tsai, Y.-T. Lyu and C.-T. Lee, Appl. Phys. Lett. 77, 687 (2000).

7. T. Hashizume, J. Appl. Phys. 94, 431 (2003).

8. I. Shalish, Y. Shalira, L. Burstein and J. Salzman, J. Appl. Phys. 89, 390 (2001).

9. T. Hashizume, S. Ootomo, S. Oyama, M. Konishi and H. Hasegawa, J. Vac. Sci. Technol. B 19, 1675 (2001).

10. D. Li, M. Sumiya, S. Fuke, D. Yang, D. Que, Y. Suzuki and Y. Fukuda, J. Appl. Phys. 90, 4219 (2001).

11. H. W. Choi, M. G. Cheong, M. A. Rana, S. J. Chua, T. Osipowicz and J. S. Pan, J. Vac. Sci. Technol. B 21, 1080 (2003).

12. J. O. Song, S.-J. Park and T.-Y. Seong, Appl. Phys. Lett. 17, 3129 (2002).

13. T. G. G. Maffeis, M. C. Simmonds, S. A. Clark, F. Peiro, P. Haines and P. J. Parbrook, J. Appl. Phys. 92, 3179 (2002).

14. K.-M. Chang, C.-C. Cheng and J.-Y. Chu, J. Electrochem. Soc. 149, G367 (2002).

15. VG Scientific Company Technical Document TD 8618.

16. J. F. Moulder, W. F. Stickle, P. E. Sobol and K. D. Bomben, Handbook of X-Ray Photoelectron Spectroscopy (Perkin-Elmer Corporation, Physical Electronics Division, USA, 1992).

17. L. Kubler, E. K. Hill, D. Bolmont and J. C. Peruchetti, Thin Solid Films 149, 385 (1987).

18. J. A. Taylor, J. Vac. Sci. Technol. 20, 751 (1982). 
Copyright of International Journal of Nanoscience is the property of World Scientific Publishing Company and its content may not be copied or emailed to multiple sites or posted to a listserv without the copyright holder's express written permission. However, users may print, download, or email articles for individual use. 